Systematic review and meta-analysis

\title{
$37 \%$ of child survivors of intrauterine or neonatal insults experience at least one long-term sequela, the most common being neurodevelopmental delay
}

\section{Anna Maria Marconi}

10.1136/eb-2012-100701

Department of Obstetrics and Gynecology, San Paolo Hospital Medical School, University of Milano, Milano, Italy

Correspondence to: Anna Maria Marconi Department of Obstetrics and Gynecology, San Paolo Hospital Medical School, University of Milano, Via A di Rudinì 8,

Milano 20142, Italy;

annamaria.marconi@unimi.it

Commentary on: Mwaniki MK, Atieno M, Lawn JE, et al. Long-term neurodevelopmental outcomes after intrauterine and neonatal insults: a systematic review. Lancet 2012;379:445-52.

\section{Implications for practice and research}

- Neonates who survive major insults in the neonatal period may suffer varying degrees of long-term neurodevelopmental impairment.

- Most insults might be prevented through primary and secondary measures such as antenatal steroid administration, use of oxygen to reduce the severity of sequelae, maternal immunisation and early detection to prevent/reduce congenital infections.

- Rehabilitation programmes for survivors should be improved by creating centres to encourage family members to participate in all aspects of care.

\section{Context}

More than $40 \%$ of mortality in children younger than 5 years occurs in the neonatal period (0-28 days), and the common causes include preterm birth complications, intrapartum-related factors, acquired infections and other conditions such as jaundice and congenital infections. ${ }^{1} 2$ Intrauterine and neonatal insults contribute to both premature mortality and long-term disability as neonates who survive major insults may suffer varying degrees of long-term neurodevelopmental impairments resulting in major socioeconomic burden, especially in resource-poor countries. However, little is known about the severity and distribution of these long-term impairments and estimates of the global burden of disease are not precisely known. Thus, the authors addressed three major questions: (1) What are the long-term outcomes after intrauterine and neonatal insults?; (2) What is the risk and severity of at least one sequela and of multiple sequelae reported after these insults? and (3) What is the risk and severity of sequelae after multiple insults for one infant?

\section{Methods}

The authors reviewed 153 studies published between 1966 and mid-2011 that met strict inclusion criteria: insults occurring during the intrauterine and neonatal period verified with appropriate diagnostic methods or criteria; follow-up for at least 6 months to exclude transient impairments; use of standardised tests for neurodevelopmental assessment; and at least $80 \%$ of neonates surviving the insult.

\section{Findings}

Overall, there were 22161 survivors followed up, with $31 \%$ survivors having one or more impairments. The most common sequelae were learning difficulties, cognition or developmental delay (59\%), cerebral palsy (21\%), hearing impairment (20\%) and visual impairment $(18 \%)$. The overall median risk of at least one sequela in any domain was $39.4 \%$. The risk was $18.5 \%$ for at least one severe impairment in any insult domain, 5\% for at least one moderate impairment and $10 \%$ for one mild impairment. For the 6558 survivors of preterm birth, $31 \%$ of infants had at least one sequela, the most frequent $(60 \%)$ being cognition, general developmental delay or learning difficulties. The risk of at least one sequela in very low birthweight newborns (1500 g or less) was $26.7 \%$. In premature newborns with sepsis, the likelihood of neurological impairment was $49 \%$ compared to $28 \%$ after preterm birth alone. Only 40 studies included data for multidomain impairments with 1048 of 2815 neonates experiencing impairments (37\%). Of these, 334 had multiple impairments and 52\% had three or more sequelae, all with cerebral palsy and learning difficulties. The pooled risk estimate of development of at least one sequela in any domain was 37\% which was the highest after rubella infection and the lowest after 
neonatal jaundice. This risk was not affected by region, duration of follow up, study design or period of data collection.

\section{Commentary}

Major strengths of this paper include strict inclusion criteria and long-term follow-up to enable assessment of neurodevelopmental outcomes. The authors acknowledge that few studies were performed in resource-poor countries, where it is difficult to collect data in nonhospital settings, and more than $98 \%$ of global neonatal morbidity occurs. Since all reviewed studies were hospital based, this might have constituted a bias in studies included from low-income countries, where patients of relatively high social and economic status have sufficient resources to access hospital care and may have lower risk of impairment.

A point of interest is that intrapartum-related events, identified as hypoxic ischaemic encephalopathy, carry a risk of $37 \%$ for at least one sequela compared to $81 \%$ for congenital rubella (the highest) and 18\% for neonatal jaundice (the lowest). In addition, many neonatal insults might be avoided through both primary and secondary prevention measures such as vaccination for rubella and tetanus, antenatal steroids and sepsis prevention for preterm birth. As 2015 approaches, it is important to ensure that the reduction in neonatal mortality (goal 4 of the Millennium Development Goals) and the improvement of maternal health (goal 5) are strictly related and together provide safe motherhood.

Competing interests None.

\section{References}

1. Lawn JE, Cousens S, Zupan J. 4 million neonatal deaths: When? Where? Why? Lancet 2005;365:891-900.

2. Lawn JE, Kinney MV, Black RE, et al. Newborn survival: a multi-country analysis of a decade of change. Health Policy Plan 2012;27(Suppl 3):iii6-28. 